\title{
COMMENTARY
}

\section{Dapsone and NETs}

\author{
Fukumi Furukawa
}

${ }^{1}$ Takatsuki Red Cross Hospital, Osaka, Japan

Keywords: dapsone; diaminodiphenyl sulfone; DDS; NETS

\section{ARTICLE INFO}

Received: February 28, 2018

Accepted: March 21, 2018

Available online: March 28, 2018

*CORRESPONDING AUTHOR

Fukumi Furukawa, Takatsuki Red

Cross Hospital, 1-1-1 Abuno,

Takatsuki, Osaka, Japan; ffurukawa@

takatsuki.jrc.or.jp

\section{CITATION}

Furukawa F. Dapsone and NETs. Trends Immunother 2018; 2(1): 634. doi: 10.24294/ti.v2.i1.634.

\section{COPYRIGHT}

Copyright (C) 2018 by author(s) and EnPress Publisher LLC. This work is licensed under the Creative Commons Attribution-NonCommercial 4.0 International License (CC BY-NC 4.0). http://creativecommons.org/licenses/ by $/ 4.0 /$
In this issue (Volume 2, Issue 1), Mikita reported an interesting case with discoid lupus erythematosus who showed transient effectiveness of dapsone for skin lesions ${ }^{[1]}$. Dapsone, also known as diaminodiphenyl sulfone (DDS) is a synthetic sulfone antimicrobials and has been used for Hansen disease as a specific medicine. In addition, dapsone is used for several dermatological disorders such as cutaneous vasculitis, systemic vasculopathy, autoimmune bullous diseases and prurigo pigmentosum ${ }^{[2]}$, in which histological neutrophil infiltration is observed in the early stage or during the course. Dapsone is thought to be central to the suppression of neutrophil function by neutrophil migration or inhibition of myeloperoxidase (MPO) and exerts the anti-inflammatory effects by interfering with the polymorphonuclear leukocyte $(\mathrm{PMN})$-dependent production of oxygen intermediates, thus conferring protection from auto-oxidative tissue injury ${ }^{[3]}$. On the other hand, neutrophil extracellular traps (NETs) have attracted attention in recent years as involvement in autoimmune diseases of neutrophils that are the immune system's first-line of defense against infection. NETs are networks of extracellular fibers, primarily composed of DNA from activated neutrophils, which bind pathogens ${ }^{[4]}$. NETs release a network structure consisting of nuclear DNA and granular proteins (MPO etc.) outside the cell to trap pathogenic microorganisms and sterilize them. Overexpression of NETs including self DNA and histone is presumed to be involved in the onset of autoimmune diseases ${ }^{[5]}$. In addition, it was reported that NETs rich in oxidized mitochondrial DNA promoted the production of type I IFN and was involved in the pathology of SLE ${ }^{[5]}$. Although the influence of dapson on NETs is unknown, NETs will provide us better understanding for considering the mechanism between dapsone and its therapeutic effect through innate immunity.

\section{References}

1. Mikita N, Furukawa F. Transient effectiveness of dapsone for skin lesions in a patient with discoid lupus erythematosus. Trends Immunother 2018; 2(1). [In press].

2. Wozel G, Blasum C. Dapsone in dermatology and beyond, Arch Dermatol Res 2014; 306(2): 103-124. doi: 10.1007/s00403-013-1409-7.

3. Miyachi Y, Niwa Y. Effects of potassium iodide, colchicine and dapsone on the generation of polymorphonuclear leukocyte-derived oxygen intermediates. $\mathrm{Br}$ J Dermatol 1982; 107(2): 209-214.

4. Brinkmann V, Reichard U, Goosmann C, et al. Neutrophil extracellular traps kill bacteria. Science 2004; 303(5663): 1532-1535. doi:10.1126/ science. 1092385.

5. Lood C, Blanco LP, Purmalek MM, et al. Neutrophil extracellular traps enriched in oxidized mitochondrial DNA are interferogenic and contribute to lupus-like disease. Nat Med 2016; 22(2): 146-153. doi: 10.1038/nm.4027. 